\title{
Methods for nanoparticle labeling of ricin and effect on toxicity
}

\author{
Alastair W. Wark*a , Jun Yu*b ${ }^{\text {b }}$, Christopher D. Lindsay ${ }^{\mathrm{c}}$, Paola Nativo ${ }^{\mathrm{a}}$, Duncan Graham ${ }^{\mathrm{a}}$ \\ ${ }^{\mathrm{a} C e n t r e}$ for Molecular Nanometrology, WestCHEM, Dept of Pure and Applied Chemistry, \\ University of Strathclyde, 295 Cathedral St, Glasgow, UK, G1 1XL; \\ ${ }^{\mathrm{b}}$ Strathclyde Institute of Pharmacy and Biomedical Sciences, University of Strathclyde, Royal \\ College, 204 George St, Glasgow, UK, G1 1XW; \\ ${ }^{c}$ Biomedical Sciences Department, DSTL, Porton Down, Salisbury, Wiltshire SP4 0JQ.
} *alastair.wark@strath.ac.uk; phone+44 (0)141 548 3084; jun.yu@strath.ac.uk; phone +44 (0)141
5482137

\begin{abstract}
The unique optical properties associated with nanostructured materials that support the excitation of surface plasmons offer many new opportunities for the enhanced optical investigation of biological materials that pose a security threat. In particular, ricin is considered a significant bioterrorism risk due to its high toxicity combined with its ready availability as a byproduct in castor oil production. Therefore, the development of optical techniques capable of rapid on-site toxin detection with high molecular specificity and sensitivity continues to be of significant importance. Furthermore, understanding of the ricin cell entry and intracellular pathways remains poor due to a lack of suitable bioanalytical techniques. Initial work aimed at simultaneously tackling both these issues is described where different approaches for the nanoparticle labeling of ricin are investigated along with changes in ricin toxicity associated with the labeling process.
\end{abstract}

Keywords: ricin, nanoparticles, labeling, toxicity, optical sensing, bioterrorism

\section{INTRODUCTION}

Ricin is a cytotoxin extracted from the seeds of castor bean plants and consists of two polypeptide chains (A and B) linked by a disulfide bond. The B-chain is a lectin which binds to cell surface receptors and facilitates transfer into the cytosol. The A-chain is an enzyme which inhibits protein synthesis by targeting intracellular 28S ribsomal RNA, where it depurinates an adenine base leading to cell death and tissue damage. ${ }^{[1-3]}$ Due to its listing as a category B bioterrorism threat, along with a heightening of security and health concerns worldwide, there has been a renewed focus on the development of sensitive and robust assays for pathogen detection in various media. However, despite extensive research into the mechanisms of ricin and other members of the AB protein toxin family, their mode of action remains poorly understood with regard to intracellular trafficking. This is partly due to a lack of bioanalytical techniques capable of detecting and tracking these toxins in real-time at the molecular level.

A number of innovative approaches to ricin detection have been reported. Traditionally, low concentrations are detected using an enzyme-linked immunosorbent assay (ELISA) or via radioactive labeling. ${ }^{[4,5]}$ However, both approaches are labor intensive and involve several time-consuming sample preparation and amplification steps which have an impact on measurement reproducibility. More recent methods include microarrays, ${ }^{[6]}$ electrochemical detection, ${ }^{[7]}$ and optical waveguides, ${ }^{[8,9]}$ all of which are based upon the specific interaction between an antibody and ricin. Other studies have focused on detecting ricin activity by monitoring inhibition of protein expression in single well devices ${ }^{[10]}$ and the measurement of adenine released from ribosomes. ${ }^{[11]} \mathrm{Gu}$ et al demonstrated that it is possible to detect single ricin molecules binding to nucleic acid aptamer-encoded nanopores fabricated in glass substrates. ${ }^{[12]}$ Also, Russell et al designed a colorimetric assay based on the controlled aggregation of carbohydrate stabilized gold nanoparticles due to 
the ability of ricin to bind simultaneously to two or more galactose molecules. ${ }^{[13]}$ Another example of the use of nanoparticles is the multiplexed detection of four toxins, including ricin, using quantum dot-antibody conjugates as part of a sandwich immunoassay. ${ }^{[14]}$

In addition to enhanced sensing opportunities, nanoparticles hold great promise for the tracking and targeted delivery of small molecules to specific organelles at the cellular level. However, there are a number of questions being asked concerning the application and biological safety of nanomaterials. For example, do nanoparticles and their possible accumulation have a toxic effect on the cell? Does their attachment to biomolecules result in changes in the cell uptake and intracellular trafficking behavior compared to that of the native ligand? Ricin enters the cell via multiple endocytic pathways and in order to reach the cytosol, where it exhibits a toxic effect, undergoes retrograde transport via the Golgi apparatus and endoplasmic reticulum. The visualization of ricin molecules at each stage of the transport process has not yet been achieved. ${ }^{[15,16]}$ Furthermore, very little information is available on the effect of nanoparticle conjugation on the ricin intracellular trafficking mechanism and toxicity.

Here, we describe work exploring different methods for the formation of gold nanoparticle -ricin conjugates. The choice of gold nanoparticles is primarily due to their unique electronic properties which can be exploited to enhance a variety of spectroscopic imaging modalities such as scattering, fluorescence and Raman. In addition to being highly sensitive to localized changes around each particle, these materials do not undergo photobleaching and offer significantly higher signal to noise than comparable measurements involving single chromophores opening up future possibilities for in-vivo ricin tracking with nanoscale spatial resolution. Initial investigations into the effect of nanoparticle labeling on toxicity compared to free ricin are also described below.

\section{EXPERIMENTAL}

Colloidal solutions of gold nanoparticles were prepared by the citrate reduction of $\mathrm{HAuCl}_{4}$. $\mathrm{Briefly}_{2} 200 \mathrm{~mL}$ of $0.25 \mathrm{mM}$ $\mathrm{HAuCl}_{4}$ was heated to boiling, after which $10 \mathrm{ml}$ of $16 \mathrm{mM}$ trisodium citrate was added under constant vigorous stirring. Both heating and stirring was continued until $30 \mathrm{mins}$ after no further color changes in the reaction solution were observed. UV-Vis and TEM characterization of the colloidal solution is shown in Figure 1(a) and (b) respectively. The average particle size is $19( \pm 4) \mathrm{nm}$. All nanoparticle concentrations reported here were calculated using an extinction coefficient of $2.4 \times 10^{8} \mathrm{M}^{-1} \mathrm{~cm}^{-1}$ associated with the intensity at the plasmon resonance peak around $520 \mathrm{~nm}$. The stock solution concentration used in the preparation of NP-ricin conjugates was $15 \mathrm{nM}$.
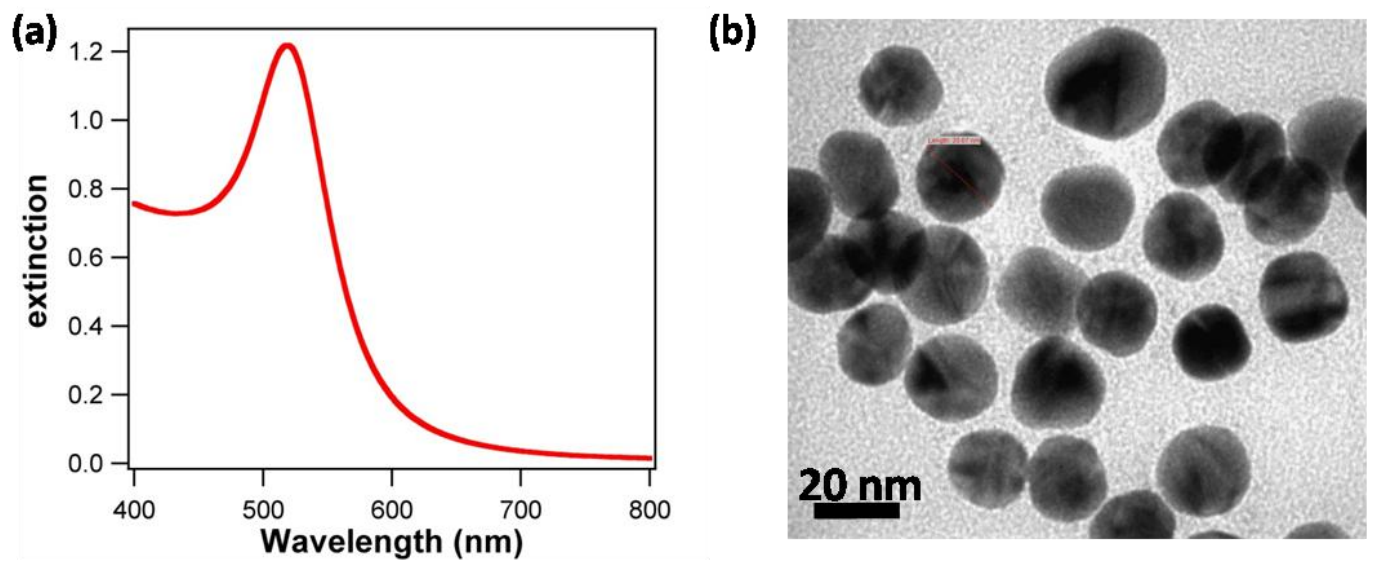

Figure 1. UV-visible spectrum (a) and TEM (b) of gold nanoparticles used in the preparation of NP-ricin bioconjugates [TEM image courtesy of Simon Smith, Detection Department, Dstl]

Ricin-nanoparticle bioconjugates were prepared using three different approaches involving either simple electrostatic attachment or by covalent coupling utilizing two different bifunctional linkers, PEG $_{41}$ and NIR-PEG . The electrostatic 
methodology relies upon the stabilizing negative charge on the nanoparticle surface. To $1 \mathrm{ml}$ of Au colloid stock, $70 \mu \mathrm{l}$ of $0.01 \mathrm{M} \mathrm{K}_{2} \mathrm{CO}_{3}$ was added followed by $10 \mu \mathrm{l}$ of $38 \mu \mathrm{M}$ ricin stock solution. After 1 hour of gentle shaking, $50 \mu \mathrm{l}$ of $5 \%$ bovine serum albumin (BSA) was added. The amount of ricin necessary for successful coating was determined from a salt titration where the minimum concentration of ricin required to prevent colloid aggregation on the addition of $10 \%$ w/w NaCl was determined. Finally, 30 mins after the addition of BSA, the samples were centrifuged at 10,000 rpm for 25 mins. The supernatant was discarded and the pellet resuspended in $10 \mathrm{mM}$ phosphate buffer, $\mathrm{pH}=7.6$. This process was repeated 4 times to remove excess ricin from the bulk solution.

The synthesis of the $\mathrm{PEG}_{41}$ and NIR-PEG 3 covalent linkers are described elsewhere. ${ }^{[17]}$ To prepare linker functionalized nanoparticles, $10 \mu \mathrm{l}$ of $10^{-3} \mathrm{M}$ linker stock solution was added to $1 \mathrm{ml}$ of Au colloid and left to incubate for a minimum of one day. The samples were then centrifuged at 10,000 rpm for 25 mins with the supernatant discarded and the resulting pellet resuspended in $10 \mathrm{mM}$ phosphate buffer, $\mathrm{pH}=7.6$. This process was performed 4 times to remove excess linker. Ricin conjugation was then performed by first adding 1-ethyl-3-(3-dimethylaminopropyl) carbodiimide (EDC), (5 $\left.\mu \mathrm{L}, 2 \mathrm{mg} \mathrm{mL}^{-1}\right)$ and $N$-hydroxysulfosuccinimide (sNHS), $\left(5 \mu \mathrm{L}, 2 \mathrm{mg} \mathrm{mL}^{-1}\right)$, to $0.5 \mathrm{~mL}$ of the linker-coated nanoparticle solution. After 15 mins, $30 \mu \mathrm{L}$ of ricin stock $(36 \mu \mathrm{M})$ was added and allowed to react for a minimum of 2 hours. Finally, excess ricin was removed by centrifuging at 10,000 rpm for $25 \mathrm{mins}$, discarding the supernatant, and resuspending the pellet in $10 \mathrm{mM}$ phosphate buffer, $\mathrm{pH}=7.6$ a total of 4 times with the final nanoparticle concentration adjusted to 10 nM.

A neutral red release assay was performed to compare the cytotoxicity of the different NP-ricin bioconjugates. Cultured MDCK epithelial cells were seeded in a 96-well bioassay plate, and incubated for 48 hours incubation at $37^{\circ} \mathrm{C}$ under $5 \%$ $\mathrm{CO}_{2}$ in Dulbecco's Modified Eagle's Medium (DMEM). Then, $250 \mu \mathrm{L}$ aliquots of free ricin, NP-ricin conjugates and linker functionalized nanoparticle controls suspended in serum-free DMEM at concentrations ranging over several orders of magnitude were added to different wells with measurements at each concentration performed in triplicate. After 16-18 hrs incubation at $37^{\circ} \mathrm{C}$ under $5 \% \mathrm{CO}_{2}$, the cells were washed twice with PBS and $200 \mu \mathrm{L}$ serum-free DMEM supplemented with neutral-red $(50 \mu \mathrm{g} / \mathrm{mL})$ was added to each well. The cells were incubated for a further 3 hours at $37^{\circ} \mathrm{C}$ under $5 \% \mathrm{CO}_{2}$, and the dye containing DMEM was removed followed by washes with PBS. The cells were then fixed with $3.7 \%$ paraformaldehyde briefly ( 5 mins), and intracellular neutral red was extracted with $1 \%$ acetic acid solution containing $30 \%$ ethanol for 20 mins at room temperature. The neutral red extracts were transferred to a fresh 96-well plate and the absorbance of neutral red at $540 \mathrm{~nm}$ was measured in each well using a Labsystems iEMS reader.

\section{RESULTS}

\subsection{Fabrication of NP-ricin conjugates}

Three different approaches were explored for the attachment of ricin to the nanoparticle surface. An overview of the different methodologies is shown in Figure 2.

The electrostatic approach for the preparation of protein-nanoparticle conjugates is well established, and traditionally employed by biologists to create specific contrast agents for use in TEM analyses of cells. The methodology relies upon the stabilizing negative surface charge associated with the citrate reduction technique for the preparation of gold and silver nanoparticles. Depending on the isoelectric point of the protein, the $\mathrm{pH}$ of the colloid solution is first increased from around 5.5 via the addition of $\mathrm{K}_{2} \mathrm{CO}_{3}$ to improve protein adsorption on to the nanoparticle surface. Typically, this process has to be optimized on an individual basis for each protein through measurement of the minimum concentration required to stabilize the colloid against salt-induced aggregation. In a second step, BSA is added to provide additional stability and reduce nonspecific nanoparticle interactions. However, it is often difficult to produce stable functionalized solutions at greater particle sizes $(>20 \mathrm{~nm}$ ) using the electrostatic method and attempts to conjugate ricin onto larger Au nanoparticles $50 \mathrm{~nm}$ in size were repeatedly unsuccessful. 


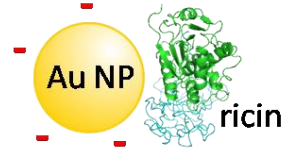

2. Covalent coupling to $\mathrm{PEG}_{41}$ linker:

Au-PEG-R<smiles>CC(COCC(C)OCC(C)NC(=O)CCCCC1CCSS12CCCCCC2)NC(=O)CCC(=O)O</smiles>

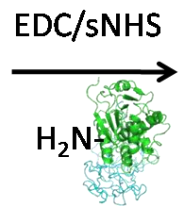

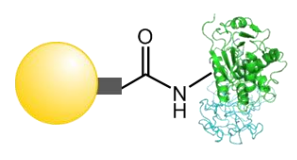

3. Covalent coupling to NIR dye-PEG 3 linker:

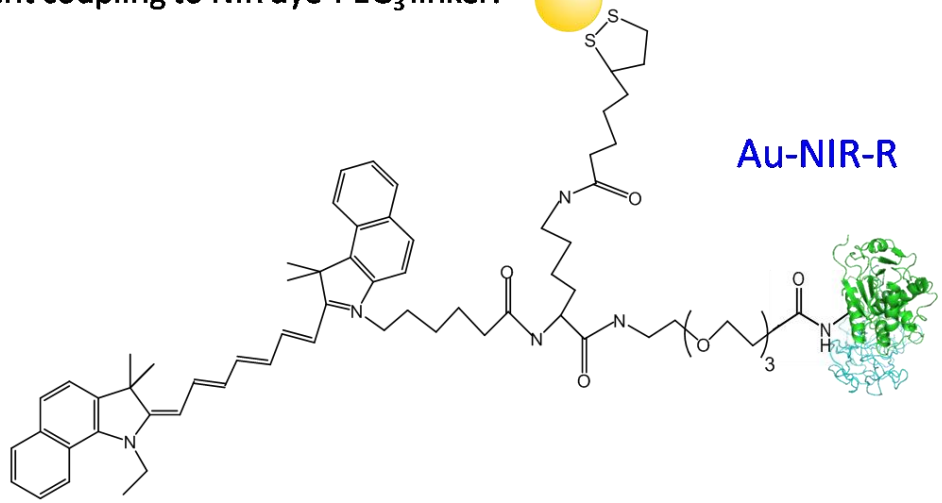

Figure 2. Overview of methodologies used for the creation of gold nanoparticle-ricin conjugates.

A more robust approach is to covalently attach ricin to the nanoparticle surface. Both the PEG $_{41}$ and NIR-PEG linkers shown in Figure 2 feature a cyclic disulfide as a surface complexing group that provides improved conjugate stability compared to mono-thiolated species. ${ }^{[18]}$ The role of the oligo(ethylene glycol) is to resist non-specific of proteins onto the nanoparticle surface as well as improving both particle solubility and stability. Typically, longer PEG lengths are required to stabilize larger nanoparticles in buffered saline solutions. Ricin attachment to the nanoparticle surface is achieved via the carboxylic acid moiety using well-established protocols for protein conjugation ${ }^{[19]}$ with the amide bond formation involving a random available primary amine on the ricin protein surface.

The NIR-PEG 3 linker in particular is part of a range of unique multifunctional nanoparticle-enhanced biomolecular labels being developed by the Graham group at the University of Strathclyde ${ }^{[17]}$ where reporter dyes of variable excitation wavelengths are incorporated within the molecular structure. These linkers can be used to create spectroscopic labels for surface enhanced resonance Raman spectroscopy (SERRS) studies which offers sensitivities equivalent or better than that of simple molecular fluorophore conjugates but with the distinct advantage of producing information-rich vibrational spectra. ${ }^{[20]}$ This provides new opportunities to simultaneously identify and track multiple analytes both in situ and in vivo.

\subsection{Confirmation of ricin conjugation}

To confirm that ricin has been successfully attached to the nanoparticle surface, a simple assay was developed where the optical sensitivity of the surface plasmon response to local changes in refractive index was utilized to measure the specific bioaffinity adsorption of anti-ricin IgG. Figure 3(a) shows the UV-visible spectrum of a prepared solution of AuNIR-R conjugate. Comparison with the spectrum in fig. 1(a) for non-functionalized Au nanoparticles indicates the conjugate to be monodisperse with the broad absorbance at longer wavelengths in figure 3(a) due to the NIR chromophore, which is only partly quenched despite its proximity to the gold nanoparticle surface. 

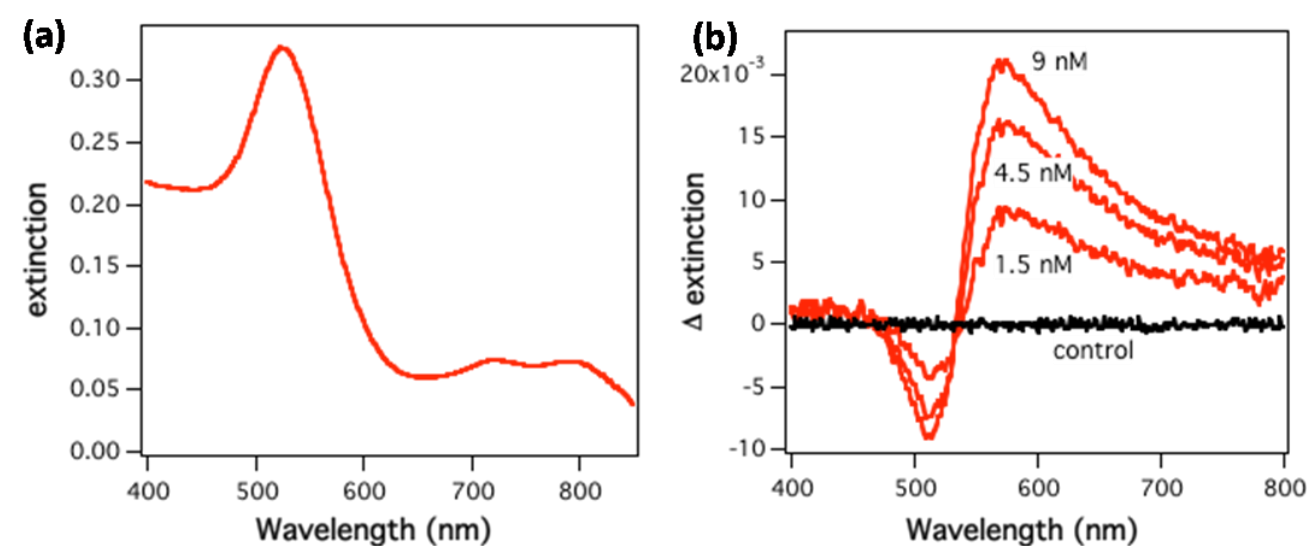

Figure 3. (a) UV-visible spectrum of Au-NIR-R conjugate in phosphate buffer solution. (b) Binding assay performed in PBS for antiricin IgG to Au-NIR-R as a function of antibody concentration. No nonspecific absorption of antibody was observed in the absence of covalently attached ricin.

Both the presence and bioactivity of conjugated ricin was confirmed by measuring changes in the extinction spectra as the bulk concentration of anti-ricin was varied from $1.5 \mathrm{nM}$ to $9 \mathrm{nM}$. Prior to performing these measurements the AuNIR-R conjugate was suspended in PBS, $\mathrm{pH}=7.4$ at a nanoparticle concentration of $1 \mathrm{nM}$. After the addition of each antibody aliquot, a final spectrum was acquired when no further changes in signal were observed. Data analysis involved first normalizing all the spectra such that each data curve has the same intensity at $450 \mathrm{~nm}$, which is within the plasmon interband transition. The differential change was then calculated by subtracting the original spectrum in the absence of antibody from each subsequent spectrum. The results in figure 3(b) clearly demonstrate the successful attachment of ricin to the nanoparticle surface using the NIR-PEG 3 linker. Similar measurements were also performed for the Au-R and Au-PEG-R conjugates with greater and smaller shifts observed respectively at equivalent antibody concentrations. This trend is in part due to lower sensitivities of the plasmon response as the antibody is spaced further from the nanoparticle surface. Furthermore, no significant antibody binding was observed on control samples where ricin had not previously been conjugated to linker functionalized nanoparticles.

\subsection{Assessing toxicity}

A series of experiments were performed to assess the effect of nanoparticle labeling and compare different attachment chemistries on ricin toxicity. An overview of the results is shown in Figure 4(a).

The neutral red assay used in this study provides a quantitative estimation of the number of viable cells in a culture and is based on the ability of viable cells to incorporate and bind the supravital dye neutral red in the lysosomes. Following overnight incubation with a range of molecular ricin and ricin-bioconjugate concentrations, the assay indicates significant differences in the minimum amount of toxin required to induce a detectable reduction in cell population with respect to the control samples. It is important to note it is nanoparticle concentration that is being reported and compared with the molecular concentration of free ricin.

The trend in reduced toxicity shown in figure 4(a) is most likely associated with an effective decrease in the nanoparticle surface coverage of ricin for the NIR-PEG 3 and $\mathrm{PEG}_{41}$ linkers. Recent attempts using an enzyme hydrolysis assay to quantify the number of proteins conjugated to the surface of similar sized gold nanoparticles via the NIR-PEG 3 linker suggest an average of less than one protein per nanoparticle. ${ }^{[21]}$ In the case of the considerably longer $\mathrm{PEG}_{41}$ linker the total number and surface density of carboxylic acid groups available for covalent coupling is expected to be significantly lower, suggesting that the ricin coupling efficiency will, in turn, be lower also. The significantly larger hydrodynamic diameter associated with the $\mathrm{PEG}_{41}$ linker may also be a factor in the rate of cell uptake. Developing an approach that enables efficient separation of nanoparticles with one or more protein molecules attached from linker-functionalized particles only has proven to be challenging and requires further work. 
(a) Minimum kill concentrations after overnight exposure:

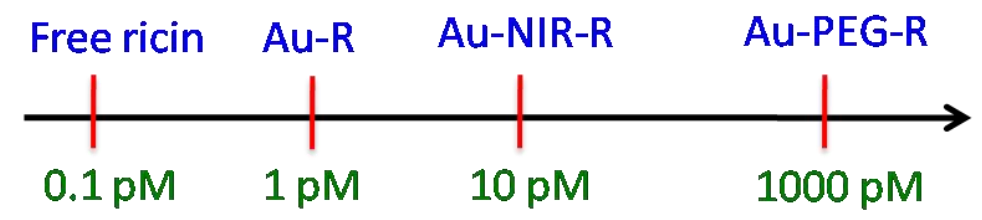

(b)

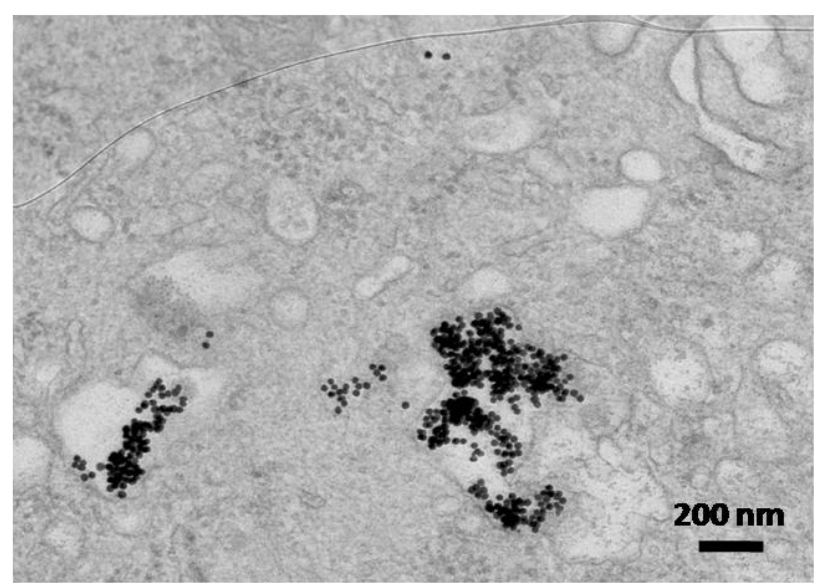

Figure 4. (a) Summary of minimum concentrations of free ricin and different nanoparticle-ricin conjugates that result in detectable cell death using a neutral red assay performed after overnight toxin incubation. (b) Representative TEM image of a transverse section of a MDCK cell after 30 mins incubation with Au-R conjugates at a concentration of $100 \mathrm{pM}$.

The uptake of nanoparticle-ricin conjugates into the cell cytoplasm was confirmed by TEM measurements. Figure 4(b) shows a sample cross-section of a MDCK cell where the sample well-plate was first exposed to a $100 \mathrm{pM} \mathrm{Au}-\mathrm{R}$ conjugate solution for only 30 mins before cell washing/fixing and TEM preparation. The image indicates receptormediated endocytosis is taking place with the presence of nanoparticle conjugates in vacuoles near the cell surface. Similar observations were made for both the Au-R and Au-NIR-R conjugates, while control experiments in the absence of attached ricin suggest a much more limited uptake, consisting of only a few isolated nanoparticles within 30 mins. Also, for Au-PEG-R, very little nanoparticle uptake was observed within this timescale. A previous TEM study ${ }^{[16]}$ examining the intracellular routing of ricin conjugated to $5 \mathrm{~nm}$ colloidal gold described marked differences between ricin-conjugate and free ricin with only the latter routed to endosomes and Golgi elements. This suggests that ricin-NP conjugates would be non-toxic and unable to inhibit protein production, which in our studies is clearly not the case. However, it is still unclear if some form of endosomal sorting takes place which results in both different intracellular pathways and uptake timescales for free and conjugated ricin molecules.

\section{CONCLUSIONS}

In this work, we have described a number of surface attachment methodologies for the preparation of ricin-nanoparticle bioconjugates. Ricin covalently attached to a nanoparticle surface was shown to remain bioactive via a simple assay involving the specific binding of anti-ricin and also via a series of toxicity studies. Future work will focus on changing the nanoparticle size and more effectively controlling the nanoparticle surface coverage orf ricin. The development of bioanalytical techniques capable of elucidating subtle differences in the uptake and intracellular response of nanoparticle-ricin conjugates compared to native ricin will be of wide importance for many other types of nanoparticleligand and cellular systems as the nanotechnology field continues to burgeon. 


\section{REFERENCES}

[1] J. M. Lord, L. M. Roberts, and J. D. Robertus, "Ricin - structure, mode of action, and some current applications," Faseb Journal, 8(2), 201-208 (1994).

[2] C. J. Marsden, D. C. Smith, L. M. Roberts et al., "Ricin: current understanding and prospects for an antiricin vaccine," Expert Review of Vaccines, 4(2), 229-237 (2005).

[3] K. Sandvig, B. Spilsberg, S. U. Lauvrak et al., "Pathways followed by protein toxins into cells," Int. J. Med. Microbiol., 293(7-8), 483-490 (2004).

[4] G. D. Griffiths, H. Newman, and D. J. Gee, "Idenitification and quantification of ricin toxin in animal-tissues using ELISA," Journal of the Forensic Science Society, 26(5), 349-358 (1986).

[5] M. A. Poli, V. R. Rivera, J. F. Hewetson et al., "Detection of ricin by colormetric and chemiluminescence ELISA," Toxicon, 32(11), 1371-1377 (1994).

[6] J. B. Delehanty, and F. S. Ligler, "A microarray immunoassay for simultaneous detection of proteins and bacteria," Anal. Chem., 74(21), 5681-5687 (2002).

[7] K. Dill, D. D. Montgomery, A. L. Ghindilis et al., "Immunoassays based on electrochemical detection using microelectrode arrays," Biosensors \& Bioelectronics, 20(4), 736-742 (2004).

[8] F. S. Ligler, M. Breimer, J. P. Golden et al., "Integrating waveguide biosensor," Anal. Chem., 74(3), 713-719 (2002).

[9] C. R. Taitt, L. C. Shriver-Lake, M. M. Ngundi et al., "Array Biosensor for Toxin Detection: Continued Advances," Sensors, 8(12), 8361-8377 (2008).

[10] Q. Mei, C. K. Fredrickson, W. Lian et al., "Ricin detection by biological signal amplification in a well-in-a-well device," Anal. Chem., 78(22), 7659-7664 (2006).

[11] M. B. Sturm, and V. L. Schramm, "Detecting Ricin: Sensitive Luminescent Assay for Ricin A-Chain Ribosome Depurination Kinetics," Anal. Chem., 81(8), 2847-2853 (2009).

[12] S. Ding, C. L. Gao, and L. Q. Gu, "Capturing Single Molecules of Immunoglobulin and Ricin with an AptamerEncoded Glass Nanopore,” Anal. Chem., 81(16), 6649-6655 (2009).

[13] C. L. Schofield, B. Mukhopadhyay, S. M. Hardy et al., "Colorimetric detection of Ricinus communis Agglutinin 120 using optimally presented carbohydrate-stabilised gold nanoparticles,” Analyst, 133(5), 626-634 (2008).

[14] E. R. Goldman, A. R. Clapp, G. P. Anderson et al., "Multiplexed toxin analysis using four colors of quantum dot fluororeagents," Anal. Chem., 76(3), 684-688 (2004).

[15] C. Tekle, B. van Deurs, K. Sandvig et al., "Cellular Trafficking of Quantum Dot-Ligand Bioconjugates and Their Induction of Changes in Normal Routing of Unconjugated Ligands," Nano Lett., 8(7), 1858-1865 (2008).

[16] B. Van Deurs, T. I. Toennessen, O. W. Petersen et al., "Routing of internalized ricin and ricin conjugates to the Golgi complex," J. Cell Biol., 102(1), 37-47 (1986).

[17] F. McKenzie, A. Ingram, R. Stokes et al., "SERRS coded nanoparticles for biomolecular labelling with wavelength-tunable discrimination," Analyst, 134(3), 549-556 (2009).

[18] J. A. Dougan, C. Karlsson, W. E. Smith et al., "Enhanced oligonucleotide-nanoparticle conjugate stability using thioctic acid modified oligonucleotides," Nucleic Acids Res., 35(11), 3668-3675 (2007).

[19] G. T. Hermanson, [Bioconjugate Techniques] Academic Press, Oxford(2008).

[20] R. J. Stokes, F. McKenzie, E. McFarlane et al., "Rapid cell mapping using nanoparticles and SERRS," Analyst, 134(1), 170-175 (2009).

[21] F. McKenzie, V. Steven, A. Ingram et al., "Quantitation of biomolecules conjugated to nanoparticles by enzyme hydrolysis," Chem. Comm.(20), 2872-2874 (2009). 